\title{
METALLICITY EFFECT ON ABSOLUTE MAGNITUDE DETERMINATION
}

\section{P. Dubois}

Observatoire de Strasbourg

In our Galaxy, it is possible to determine the absolute magnitude of a star by mean of the intensity of the hydrogen lines, if we know the spectral type of the star or its temperature (see for instance Petrie, 1965; Hutchings, 1966; Balona and Crampton, 1974; Crawford, 1973, 1978; ...).

$$
\mathrm{M}_{\mathrm{v}}=\mathrm{f}\left(\mathrm{W}_{\mathrm{H}} \text {, Sp.T. }\right)
$$

Such a relation (full lines) is represented in the lower part of the fig. 1 for galactic cluster stars (triangles). The absolute magnitude of a star is obtained from the distance modulus of the cluster corrected by the mean absorption of the cluster. The distance moduli are only determined by fitting the main sequence with the ZAMS. A diagram can be obtained for the Small Magellanic Cloud also (upper part of the fig. 1 - dotted lines) taking into account the apparent visual magnitude of the stars (circles) corrected for the absorption of the stars and a mean distance modulus of 19.0 magnitude for the SMC. The photometric data are from Azzopardi and Vigneau (1975) and the spectroscopic data from Dubois et al. (1977) and Dubois (1982).

We observe that the two sets of relations are not the same. The temperature effect is much higher in the SMC stars than in the galactic ones.

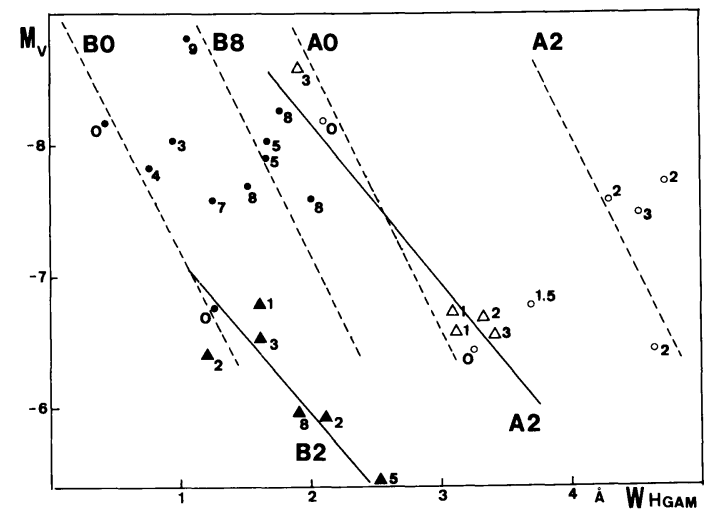

Figure 1. Comparison of the SMC relation (dotted lines) with the galactic one (full lines). Numbers are subclasses of spectral types. 
This means that the hydrogen lines are generally stronger in the SMC than in the galactic ones. We may suspect errors in the equivalent width, but many verifications have been made which exclude such an explanation. A similar effect can be seen in the data of Hutchings (1966). Moreover, the photometric narrowband index H-beta gives similar results with the data of Osmer (1973).

If we use only a restricted range in spectral type to make the fit of the two calibrations, in order to obtain the distance modulus of the SMC, we obtain different distance moduli as a function of the mean spectral observed in the SMC and in the reference stars. The results are presented in table 1 for the preceding calibrations.

Table 1. Distance modulus of the SMC as a function of the mean spectral type used.

\begin{tabular}{lcccccc}
\hline & \multicolumn{3}{c}{ H-gamma } & \multicolumn{3}{c}{ H-beta } \\
Gal. & B2 & A0 & A2 & B2 & A0 & A2 \\
\hline SMC & & & & & & \\
B0 & 19.3 & & & 20.0 & & \\
B3 & 18.5 & & & 19.5 & & \\
B5 & & & 19.0 & & \\
B8 & 17.6 & & 18.0 & 19.2 & \\
A0 & & 19.0 & & & 18.4 & \\
A2 & & 16.8 & & & & 16.8 \\
\hline
\end{tabular}

This effect could explain that some spectroscopic distance moduli of the SMC were too small compared with those determined by other methods (Buscombe and Kennedy, 1962; Westerlund et al., 1963; Hutchings, 1966).

Previous mentions of abnormal strong hydrogen lines in the Magellanic Cloud stars have been given by Fehrenbach and Duflot (1972), van den Bergh (1976) or Humphreys (1983). Does this come from the small metal content of the SMC ? It is one of the first explanation -see for instance Tully and Wolff (1984)- but no atmospheric model has yet explained such a difference in the stellar spectra.

\section{REFERENCES}

Azzopardi, M. and Vigneau, J. : 1975, Astron. Astrophys. Suppl. Ser. 22, 285 Balona, I. and Crampton, D. : 1974, Mon. Not. Roy. Astron. Soc. 166, 203 Buscombe, W. and Kennedy, P.M. : 1962, J. Roy. Astron. Soc. Canada 56, 113 van den Bergh, B. : 1976, IAU Coll. no.37, p. 13

Crawford, D.L. : 1973, IAU Symp. no. 54, p. 93

Crawford, D.L. : 1978, Astron. J. 83, 48

Dubois, P., Jaschek, M. and Jaschek, C. : 1977, Astron. Astrophys. 60, 205

Dubois, P. : 1982, Astron. Astrophys. Suppl. Ser. 48, 375

Fehrenbach, Ch. and Duflot, M. : 1972, Astron. Astrophys. 21, 231

Humphreys, R.M. : 1983, Astrophys. J. 265, 176

Hutchings, J.B. : 1966, Mon. Not. Roy. Astron. Soc. 132, 433

Petrie, R.M. : 1965, Publ. Dom. Astrophys. Obs. 12, 317

Tully, R.B . and Wolff, S.C. : 1984, Astrophys. J. 281, 67

Westerlund, B.E., Danziger, I.J. and Graham, J. : 1963, Observatory 83, 74 